\title{
Electrical stimulation counteracts muscle decline in seniors
}

\section{Helmut Kern ${ }^{1,2+}$, Laura Barberi ${ }^{3 \dagger}$, Stefan Löfler ${ }^{2}$, Simona Sbardella ${ }^{3}$, Samantha Burggraf ${ }^{2}$, Hannah Fruhmann ${ }^{2}$, Ugo Carraro ${ }^{2,4}$, Simone Mosole ${ }^{2,4}$, Nejc Sarabon ${ }^{5}$, Michael Vogelauer ${ }^{1}$, Winfried Mayr ${ }^{6}$, Matthias Krenn ${ }^{6}$, Jan Cvecka ${ }^{7}$, Vanina Romanello ${ }^{8}$, Laura Pietrangelo ${ }^{9}$, Feliciano Protasi ${ }^{9}$, Marco Sandri $^{8}$, Sandra Zampieri ${ }^{2,10}$ and Antonio Musaro ${ }^{3,10 *}$}

1 Institute of Physical Medicine and Rehabilitation, Wilhelminenspital, Vienna, Austria

${ }^{2}$ Ludwig Boltzmann Institute of Electrical Stimulation and Physical Rehabilitation, Vienna, Austria

${ }^{3}$ DAHFMO-Unit of Histology and Medical Embryology, Institute Pasteur Cenci-Bolognetti, IIM, Sapienza University of Rome, Rome, Italy

${ }^{4}$ Laboratory of Translation Myology, Department of Biomedical Sciences, University of Padova, Padova, Italy

${ }^{5}$ Science and Research Centre, Institute for Kinesiology Research, University of Primorska, Koper, Slovenia

${ }^{6}$ Center of Medical Physics and Biomedical Engineering, Medical University of Vienna, Vienna, Austria

7 Faculty of Physical Education and Sport, Comenius University, Bratislava, Slovakia

${ }^{8}$ Dulbecco Telethon Institute at Venetian Institute of Molecular Medicine, Padova, Italy

${ }^{9}$ CeSI-Center for Research on Aging \& DNICS - Department of Neuroscience, Imaging and Clinical Sciences, University G. d'Annunzio of Chieti, Chieti, Italy

${ }^{10}$ Center for Life Nano Science@Sapienza, Istituto Italiano di Tecnologia, Rome, Italy

\section{Edited by:}

Luciano Merlini, Istituto Ortopedico

Rizzoli IRCCS, Italy

Reviewed by:

Maurilio Sampaolesi, University of Leuven, Belgium

Rüdiger Rudolf, University of Applied

Sciences Mannheim, Germany

*Correspondence:

Antonio Musaro, Unit of Histology and Medical Embryology, Via A.

Scarpa 14, Rome 00161, Italy

e-mail: antonio.musaro@uniroma1.it

${ }^{+}$Helmut Kern and Laura Barberi have contributed equally to this work.

The loss in muscle mass coupled with a decrease in specific force and shift in fiber composition are hallmarks of aging. Training and regular exercise attenuate the signs of sarcopenia. However, pathologic conditions limit the ability to perform physical exercise. We addressed whether electrical stimulation (ES) is an alternative intervention to improve muscle recovery and defined the molecular mechanism associated with improvement in muscle structure and function. We analyzed, at functional, structural, and molecular level, the effects of ES training on healthy seniors with normal life style, without routine sport activity. ES was able to improve muscle torque and functional performances of seniors and increased the size of fast muscle fibers. At molecular level, ES induced up-regulation of IGF-1 and modulation of MuRF-1, a muscle-specific atrophy-related gene. ES also induced up-regulation of relevant markers of differentiating satellite cells and of extracellular matrix remodeling, which might guarantee shape and mechanical forces of trained skeletal muscle as well as maintenance of satellite cell function, reducing fibrosis. Our data provide evidence that ES is a safe method to counteract muscle decline associated with aging.

Keywords: electrical stimulation, aging, muscle performance, muscle atrophy, IGF-1, extracellular matrix, satellite cells, microRNA

\section{INTRODUCTION}

There is considerable clinical interest in therapeutic strategies to counteract muscle wasting associated with aging.

Skeletal muscle is particularly susceptible to the effects of aging, undergoing a steady reduction in function and losing up to a third of its mass and strength. This decline in functional performance is due to an overall decrease in muscle integrity, as fibrosis and fat accumulation replace functional contractile tissue, and to loss of the fastest most powerful fibers (Scicchitano et al., 2009; Vinciguerra et al., 2010).

At present, it is clear that the most efficient method that has been used to counteract age-related muscle weakness is long term physical exercise (Paffenbarger et al., 1994). Physical exercise increases protein synthesis, turnover and satellite cell number, stimulates appetite, increases IGF-1 expression levels, and capillary bed density. We recently reported that physical exercise in seniors preserves muscle morphology and ultrastructure, guarantees a greater maximal isometric force and function, and modulates the expression of genes related to autophagy and reactive oxygen species detoxification (Mosole et al., 2014; Zampieri et al., 2014). Nevertheless, certain pathologic conditions and aging limit the effectiveness of exercise and, therefore, the benefits from it.

An alternative effective intervention to improve muscle recovery is electrical stimulation (ES) (Quittan et al., 2001; Nuhr et al., 2004; Bax et al., 2005; Strasser et al., 2009). ES has been used in clinical settings for rehabilitation purposes, as an alternative therapeutic approach to counteract neuromuscular disability, as well as for muscle strengthening and maintenance of muscle mass in seniors (Maddocks et al., 2013). In addition, there are studies showing that patients with knee osteoarthritis can benefit from the use of ES alone or as an adjunct therapy (Rosemffet et al., 2004; Levine et al., 2013). ES directly stimulates skeletal muscle protein synthesis rates (Wall et al., 2012). Although controversial results have been published as consequence of varying protocols (e.g., training 3-7 times a week, training period from 3-12 weeks) and stimulation parameters (e.g., stimulation duration 2-30 s, stimulation frequency 8-80 Hz) (Giggins et al., 2012; de Oliveira Melo et al., 2013), ES represents a promising adjuvant treatment to attenuate muscle disability. Nevertheless, the molecular mechanisms by which ES exerts its specific anabolic effects on skeletal muscle remain to be elucidated. 
Based on our documented clinical experience on the use of ES to rescue permanently denervated skeletal muscles in paraplegics (Kern et al., 2004, 2008, 2010; Ashley et al., 2007; Boncompagni et al., 2007), we verified whether ES can be proposed as a therapeutic tool to rehabilitate skeletal muscle of sedentary seniors.

We demonstrated that ES mimics the beneficial effects of physical exercise in muscle of aging individuals and we defined the molecular signature underlying these effects.

\section{MATERIALS AND METHODS SUBJECTS ENROLLED IN THE STUDY}

Sixteen subjects (eight male and eight female) (73.1 \pm 6.9 years, $81.7 \pm 14.7 \mathrm{~kg}, 170.3 \pm 11.2 \mathrm{~cm}$ ) were recruited for the study. All of the subjects were volunteers who signed an informed consent and received detailed information about the functional test protocols, the trainings, and muscle biopsies. Approval from the national committee for medical ethics was obtained at the beginning of the study (EK08-102-0608). All subjects included were healthy and declared not to have any specific physical/disease issue and were instructed to maintain their normal daily activities during the training period. Various functional tests, force measurement, and muscle biopsy were performed twice, namely 1 week before and 1 week after 9 weeks of ES training.

\section{ELECTRICAL STIMULATION TRAINING}

Subjects were exposed to regular neuromuscular ES training (swelling current) for a period of 9 weeks, starting two times a week for the first 3 weeks and then switched to three times a week for the next 6 weeks, amounting to a total of 24 training sessions $(3 \times 10$ min each session). ES training was performed with a two channel custom-built battery-powered stimulator (Krenn et al., 2011) at home by the subjects themselves after detailed instructions. The subjects applied two conductive rubber electrodes $\left(9 \mathrm{~cm} \times 14 \mathrm{~cm} ; 126 \mathrm{~cm}^{2}\right)$, which were attached to the skin by wet sponge on the anterior thigh on both sides (left/right). The electrode pairs for left and right thigh were connected to the two channels of the stimulator. This allowed independent activation of the left and right thigh muscles, which were stimulated in an alternative manner. Each repetition (i.e., ES evoked muscle contraction) was evoked by a $3.5 \mathrm{~s}$ train $(60 \mathrm{~Hz})$ of electrical pulses (rectangular, biphasic, width $0.6 \mathrm{~ms}$ ). Consecutive contractions of the same thigh were separated by 4.5 s intervals. In this study, constant voltage stimulation devices were applied. The subjects were instructed to increase the stimulation intensity until their maximum sensory tolerance level was reached. With this intensity all of the subjects achieved full knee extension. Nevertheless, the applied current and voltage was recorded by the stimulation device for each training session. The mean stimulation current was $128 \pm 16 \mathrm{~mA}$ and voltage of $39 \pm 14 \mathrm{~V}$.

\section{FORCE MEASUREMENT}

An isometric measurement on a dynamometer (S2P Ltd., Lubljana, Slovenia) as described (Šarabon et al., 2013a,b) with $90^{\circ}$ hip flexion and $60^{\circ}$ knee flexion (full knee extension $=0^{\circ}$ ) was performed three times at each leg to assess the maximal isometric torque of the left and right knee extensors. The mean of the best values of each leg were taken for further analyses.

\section{FUNCTIONAL TESTS}

A complete set of functional tests to access mobility and function in activities of daily living (ADL) was designed and applied to each of the subjects. These tests included: time up and go test (TUGT) (Podsiadlo and Richardson, 1991) where the subjects were asked to stand up from a standard chair, walk a distance of $3 \mathrm{~m}$, turn around, walk back to the chair, and sit down again all as fast as possible; short physical performance battery (SPPB) (Guralnik et al., 1994) to evaluate the lower extremities function by using tests of gait speed $(2.4 \mathrm{~m})$, standing balance (side-by-side, semi-tandem, and tandem stance for $10 \mathrm{~s}$ ) and the time which the subject needed to rise from a chair for five times as quickly as possible with the arms folded across their chest; 12 flight Stair Test (Suzuki et al., 2001) where the participant was instructed to ascend and descend the stairs after reaching the top (12th) step as quick and safe as possible; and $10 \mathrm{~m}$-walking test with habitual and fastest walking speed (but not running) (Šarabon et al., 2010a,b) where each speed was performed three times, the time was measured and average velocity calculated.

\section{MUSCLE BIOPSIES}

Muscle biopsies were harvested as described (Kern et al., 2004) from the vastus lateralis muscle $15-20 \mathrm{~cm}$ proximal of the joint space of the knee, with the Bergström needle inserted perpendicular to the fiber direction. The biopsies before training were taken 10 days after the initial assessment at inclusion to the study, ES training started 14 days later. Post-training biopsies were taken 7 days after the last training session. The final functional assessment was done 4 days after the last training session. About $50-70 \mathrm{mg}$ of tissue was harvested from both legs of the subjects.

\section{Histological analysis}

For light microscopy analyses, serial cryosections ( $8 \mu$ thick) from frozen muscle biopsies were mounted on polysine ${ }^{\mathrm{TM}}$ glass slides, air-dried, and stained either with Hematoxylin-Eosin (HE) or for myofibrillar ATPases to evaluate muscle fiber type using conventional techniques as described (Rossini et al., 2002). Slowtype muscle fibers are dark-stained, while the fast-type fibers are light-stained following pre-incubation at $\mathrm{pH} 4.35$.

\section{Morphometric analysis}

The mean myofiber diameter and the percentage of slow and fasttype muscle fibers were evaluated from stained cross sections in accordance with our previous published methods (Rossini et al., 2002; Carraro et al., 2005; Ashley et al., 2007; Biral et al., 2008; Kern et al., 2008, 2010). Images were acquired using a Zeiss microscope connected to a Leica DC300F camera. Morphometry analysis was performed using Scion Image software (2000 Scion Corporation, Inc.).

\section{IMMUNOFLUORESCENCE ANALYSIS}

Muscle sections were incubated either for $1 \mathrm{~h}$ at room temperature (RT) or overnight at $4^{\circ} \mathrm{C}$, with anti-neural adhesion molecule (N-CAM) rabbit polyclonal antibody (Chemicon, Italy), anti-Pax7 mouse monoclonal antibody (DSHB, Iowa), or anti-laminin rabbit polyclonal antibody (Sigma, Italy) 1:100 diluted in PBS, respectively, as described (Zampieri et al., 2010; Mosole et al., 2014). 
Sections were then incubated for $1 \mathrm{~h}$ at RT with $\mathrm{Cy} 3$ or Alexa Fluor ${ }^{\circledR} 488$ dye conjugated antibodies against rabbit (Chemicon, Italy) or mouse IgG (Life technologies, Italy). Sections were then mounted on glass slides using ProLong Gold antifade reagent with DAPI (Life Technologies). Quantitation of Pax7 positive cells were performed on captured images from random fields counting a minimum of 300 fibers per biopsy.

\section{GENE EXPRESSION ANALYSES AND miRNA}

Total RNA extraction from human muscle biopsies before and after ES was performed with tissue lyser (Qiagen) in TriRiagentTM (Sigma) and small RNAs were purified using PureLink miRNA Isolation Kit (Invitrogen). This RNA fraction, containing microRNA (miRNA), was reverse-transcribed using the TaqMan ${ }^{\circledR}$ MicroRNA Reverse Transcription Kit (Life Technologies); the other RNA fraction, containing mRNA, was reverse-transcribed using a QuantiTect Reverse Transcription kit (Qiagen). The reverse-transcription reactions were performed according to the manufacturers' instructions. Quantitative PCR was performed on an ABI PRISM 7500 SDS (Applied Biosystems, USA), using pre-made 6-carboxyfluorescein (FAM)-labeled TaqMan assays for GAPDH, IGF-1 Ea, IGF-1 Eb, IGF-1 Ec, IGF-1 pan, Myostatin, Collagen I, III, VI (Applied Biosystems, USA). FAM-labeled TaqMan MicroRNA Assays for miR-1, miR-133a, miR-206, miR-29, and U6 snRNA (Applied Biosystems, USA) were performed as described. Quantitative RT-PCR sample values were normalized to the expression of GAPDH mRNA or U6 snRNA. The relative level for each gene and miRNA was calculated using the 2-DDCt method (Livak and Schmittgen, 2001) and reported as mean fold change in gene expression.

\section{STATISTICAL ANALYSES}

SPSS Statistics software package, version 17.0 was used to evaluate differences between the measurements in parameters of torque, functional tests, muscle morphometry, and molecular data. Normal distribution was obtained with Shapiro-Wilk-Test, the twotailed paired and unpaired Student's $t$-test and Wilcoxon-Test were used for normal and not normal distributed variables, respectively. For differences presented in percentage the 95\% confidence interval (CI) was calculated. The level of significance was set to $p<0.05$.

\section{RESULTS \\ ELECTRICAL STIMULATION IMPROVES FUNCTIONAL PERFORMANCES AFTER 9 WEEKS OF TRAINING}

To assess mobility, frailty, and risk of falling, behavior analyses in challenging conditions as TUGT and SPPB is recommended (Freiberger et al., 2013; Viana et al., 2013).

With ES training, we improved (i.e., shortened) the TUGT time $(-16.4 \% \pm 6.1 \mathrm{CI}, p<0.0005)$ and increased the SPPB Score $(+11.2 \% \pm 6.8 \mathrm{CI}, p<0.005)$ (Table 1$)$, resulting in a greater mobility in seniors recruited for this study.

For older adults, the ability to rise from a chair and sit down five times consecutively is a parameter to measure the degree of independence (Corrigan and Bohannon, 2001; Freiberger et al., 2013) and is considered as an index of muscle strength
(Bohannon, 1997). Since the test is specific to lower body strength and power, the significant pre-post-test improvement $(-23.9 \% \pm 8.6 \mathrm{CI}, p<0.005)$ of the $5 \times$ Chair Rise Test indicates a sufficient training effect of ES (Table 1).

The maximum isometric torque, an important factor for gait and physical function and a key factor against sarcopenia (CruzJentoft, 2013) developed by the Quadriceps $(+6.0 \% \pm 4.9 \mathrm{CI}$, $p<0.05$ ), was significantly improved by ES training (Table 1).

The ability to climb stairs in a secure and fast manner is an essential eccentric and concentric strength performance of the lower extremities in daily life (Rejeski et al., 1995). The significant decrease of stair test time $(-21.1 \% \pm 10.8 \mathrm{CI}, p<0.05)$ in our ES-treated subjects indicates a greater performance and safety for the ADL (Table 1).

Gait speed is relevant to the functioning of seniors in the community and an important predictor for the onset of disability, commonly used by physical therapists and other clinicians (Guralnik et al., 2000; Bohannon and Williams Andrews, 2011) and known as good predictor for frailty (Cruz-Jentoft, 2013; Viana et al., 2013). The significant increase of the $10 \mathrm{~m}$ test habitual as well as fastest walking speed $(+5.3 \% \pm 4.6 \mathrm{CI}, p<0.05$ and $+4.9 \% \pm 3.7 \mathrm{CI}, p<0.05$, respectively) supports the functional changes and are good indicators of prevention of frailty and falls (Table 1).

\section{ELECTRICAL STIMULATION MAINTAINS MUSCLE MASS AND ENHANCES SATELLITE CELLS ACTIVATION, PROMOTING MUSCLE ADAPTATION}

We also monitored whether the aforementioned functional benefit, exerted by ES, was associated with a morphological gain. ES training maintained the overall mean myofiber diameter (Figures 1A,B; Table 2), while significantly increased the diameter of fast-type myofibers and decreased that of slow fibers type (Figures 1C,D; Table 2). Changes in fiber-type distribution were also observed, even though not significantly (Table 2).

Of note, no sign of fibrosis and/or inflammatory cell infiltration was detected in treated muscles (Figure 1). Moreover, ultrastructural analysis did not reveal alterations in muscle structure between pre- and post-trained muscles, nor differences in the frequency and position of calcium release units (CRUs) and mitochondria between the two groups of samples (data not shown).

A critical role in muscle homeostasis and regeneration is exerted by satellite cells (Carosio et al., 2011), which can be also activated by different stimuli, including physical exercise (Kadi et al., 1999; Snijders et al., 2009; Walker et al., 2012).

To verify whether ES promotes a similar response of exercise, we analyzed the expression of relevant molecular markers of activated and committed satellite cells such as N-CAM, Pax7, and myogenin (Carosio et al., 2011). Immunofluorescence analysis revealed that ES induced a significant increase in the percentage of N-CAM (Figure 2A) and Pax7 (Figure 2B left and right panels) expressing cells, along with a significant increase in myogenin expression, analyzed by RT-PCR analysis (Figure 2C). Recent studies have shown that muscle cell proliferation and differentiation are mediated by a collection of muscle-specific miRNAs (van Rooij et al., 2008). miR-206 is expressed in early phases of differentiation, 
Table 1 | Force measurements and functional tests of seniors with muscle weakness before and after ES training.

\begin{tabular}{|c|c|c|c|c|}
\hline & Pre & Post & Improvement & $t$-test \\
\hline \multicolumn{5}{|l|}{$\operatorname{ALL}(N=16)$} \\
\hline Torque (Nm/kg) & $1.42 \pm 0.34$ & $1.51 \pm 0.38$ & $6.0 \pm 4.9$ & $p<0.05$ \\
\hline $5 \times$ chair rise $(s)$ & $13.85 \pm 3.33$ & $10.53 \pm 3.63$ & $-23.9 \pm 8.6$ & $p<0.005^{a}$ \\
\hline SPPB score & $10.06 \pm 1.39$ & $11.19 \pm 1.22$ & $11.2 \pm 6.8$ & $p<0.005^{a}$ \\
\hline $10 \mathrm{~m}$ test fast $(\mathrm{m} / \mathrm{s})$ & $1.58 \pm 0.28$ & $1.66 \pm 0.24$ & $4.9 \pm 3.7$ & $p<0.05$ \\
\hline \multicolumn{5}{|l|}{ FEMALE $(N=8)$} \\
\hline Torque (Nm/kg) & $1.35 \pm 0.32$ & $1.45 \pm 0.37$ & $7.5 \pm 7.4$ & 0.058 \\
\hline TUGT (s) & $9.13 \pm 1.82$ & $7.58 \pm 0.96$ & $-16.9 \pm 9.0$ & $p<0.05$ \\
\hline $5 \times$ chair rise (s) & $13.52 \pm 3.30$ & $9.01 \pm 1.19$ & $-33.3 \pm 12.5$ & $p<0.005$ \\
\hline \multicolumn{5}{|l|}{ MALE $(N=8)$} \\
\hline Torque (Nm/kg) & $1.50 \pm 0.36$ & $1.57 \pm 0.41$ & $4.5 \pm 6.7$ & $0.208^{a}$ \\
\hline TUGT (s) & $7.71 \pm 1.92$ & $6.49 \pm 0.97$ & $-15.8 \pm 8.8$ & $p<0.05$ \\
\hline $5 \times$ chair rise $(s)$ & $14.22 \pm 3.60$ & $12.28 \pm 4.74$ & $-13.7 \pm 9.4$ & $p<0.05$ \\
\hline SPPB score & $10.0 \pm 1.31$ & $10.63 \pm 1.51$ & $6.3 \pm 3.6$ & $p<0.05$ \\
\hline Stair test (s) & $14.92 \pm 4.37$ & $12.69 \pm 2.77$ & $-15.0 \pm 13.1$ & 0.161 \\
\hline $10 \mathrm{~m}$ test habitual $(\mathrm{m} / \mathrm{s})$ & $1.31 \pm 0.16$ & $1.35 \pm 0.17$ & $3.5 \pm 3.9$ & 0.125 \\
\hline $10 \mathrm{~m}$ test fast $(\mathrm{m} / \mathrm{s})$ & $1.74 \pm 0.29$ & $1.81 \pm 0.22$ & $3.5 \pm 4.0$ & 0.173 \\
\hline
\end{tabular}

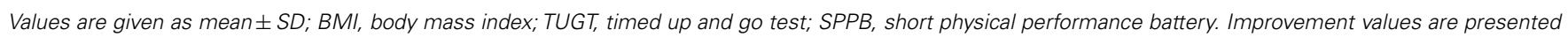
as difference in percentage $\pm 95 \%$ confidence interval.

Wilcoxon-Test.

whereas the expression of miR-1 is a marker of terminal differentiation and controls the expression of relevant enzymes in the response to oxidative stress (Chen et al., 2006; Rao et al., 2006; Cacchiarelli et al., 2010). Real time PCR analysis (Figure 2C) revealed a significant up-regulation of miR-206 and an increase of miR-1 expression in ES stimulated muscle compared to control muscle.

\section{CHARACTERIZATION OF MOLECULAR PATHWAYS INVOLVED IN ES-MEDIATED MUSCLE ADAPTATION}

To determine the adaptation changes of gene expression due to ES, we performed RT-PCR to quantify shifts in mRNA levels of a selected panel of genes involved in muscle growth and plasticity, in pre-trained (used as control) and electrical stimulated (treated) aged muscles. One of the key factors involved in skeletal muscle adaptations and growth is insulin-like growth factor-1 (IGF-1) (Musarò et al., 2001; Berg and Bang, 2004; Adamo and Farrar, 2006; Scicchitano et al., 2009; Kern et al., 2011).

We analyzed the expression of the different isoforms of IGF-1. In humans, three mRNA variants (known as IGF-1Ea, IGF-1Eb, and IGF-1Ec) with alternatively spliced ends have been identified (Scicchitano et al., 2009; Vinciguerra et al., 2010). Figure 3A shows that $\mathrm{ES}$ promoted a significant increase in the mRNA expression of total (pan) IGF-1 and of IGF-1Ea, IGF-1Eb, and IGF-1Ec isoforms.

To verify whether ES stimulates not only anabolic pathways, but negatively modulates muscle catabolism, we analyzed the expression of factors associated with relevant proteolytic systems such as the ubiquitin-proteasome and the autophagylysosome systems (Vinciguerra et al., 2010). Atrogin-1 and MuRF1 are muscle-specific atrophy-related ubiquitin ligases and are responsible for the increased protein degradation through the ubiquitin-proteasome system (Vinciguerra et al., 2010). We found a significant down-regulation of MuRF-1 and a reduced trend in atrogin-1 expression in the post-training group (Figure 3B). The autophagy-related genes Beclin 1, Bnip3, and p62 did not change in trained muscles indicating that ES do not modulate the autophagy pathway (Figure 3B).

Another key modulator of muscle mass is myostatin (Elliott et al., 2012). Myostatin has been described as a negative regulator of skeletal muscle mass and regeneration and a target of miR-206 (Clop et al., 2006). Surprisingly, real time PCR revealed an upregulation of myostatin mRNA in ES-treated muscle compared to control pre-trained muscle (Figure 3C).

The age-related decrease in muscle mass involves a selective loss of fast glycolytic fibers (Type II) over slow oxidative fibers (Type I) (Alnaqeeb and Goldspink, 1987). Although the Type I fibers are energetically more efficient than Type II fibers, so that senescent muscle should become progressively more resistant to fatigue, they are also greatly decreased in their force-generating capacity, exhibiting restricted contractile options in terms of speed and power output. Since ES involved an improvement in 
A

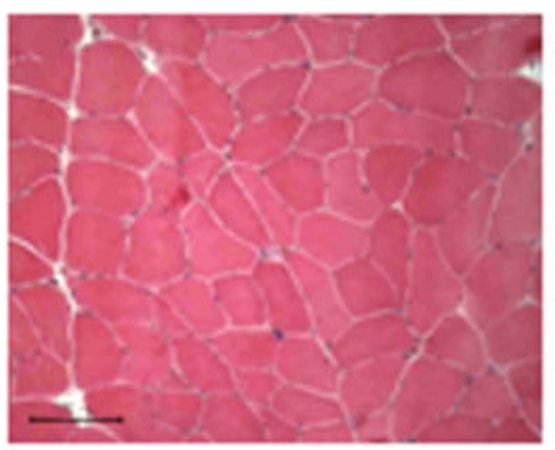

C

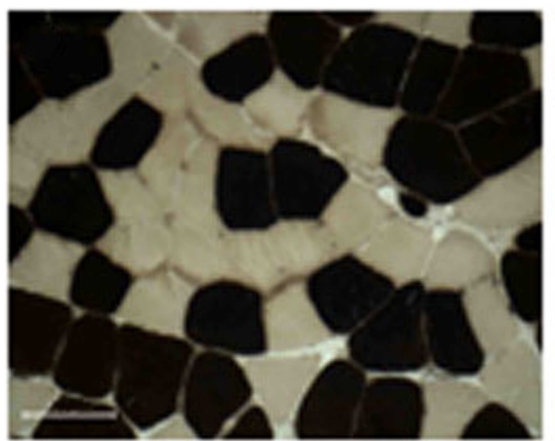

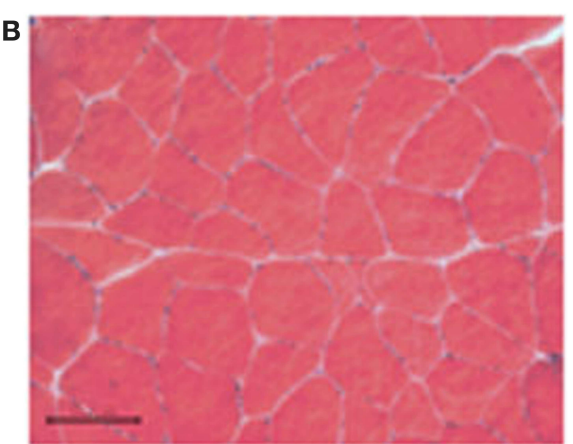

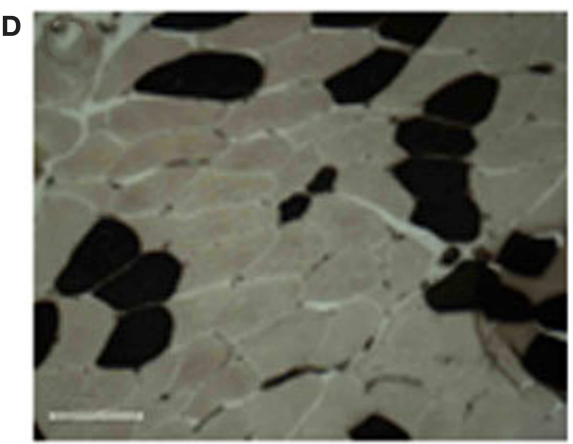

FIGURE 1 | Muscle morphology and fiber-type distribution. All muscle biopsies present well-packed myofibers, without signs of fibrosis, and inflammatory cell infiltration before (A) or after 9 weeks of training (B). The training induced an increase of either diameter and percentage of the fast-type fibers [brown stained (C,D)]. Bar $100 \mu \mathrm{m}$

muscle strength and power, we analyzed one of the key factors involved in oxidative metabolism and fiber-type switching, namely PGC1 $\alpha$ (Lin et al., 2002). Of note, PGC1 $\alpha$ was down-regulated in ES-treated muscles (Figure 3D), indicating a maintenance of the fastest more powerful phenotype. Since down-regulation of PGC1 $\alpha$ could point to altered mitochondrial function and therefore to potential increase in ROS production, we monitored the expression of Nrf2, a transcription factor that has a central role in oxidative stress response in worms, flies, and mice. Importantly, expression of the gene Nrf2 did not change with ES (Figure 3E).

Interestingly, muscle extracellular matrix (ECM) constitutes a vital adaptation in providing protection against contractioninduced injury in human skeletal muscle (Mackey et al., 2011).

To support this hypothesis, we analyzed, by real time PCR, the expression of adhesion-promoting matrix components, demonstrating a significant up-regulation of collagen types I and III in ES muscle compared to control pre-trained muscle (Figure 4). Of note, ECM represents also a niche component of satellite cells. One of the matrix components that might play a role in maintaining satellite cell function is collagen VI (Urciuolo et al., 2013). Real time PCR analysis revealed a significant increase in Collagen VI expression in ES-trained muscle compared to pre-trained muscle (Figure 4).

Interestingly, the up-regulation of ECM regulators was not associated with accumulation of fibrotic tissue, as revealed by histological analysis (Figure 1), suggesting that ECM remodeling is indeed an important homeostatic event promoted by ES.
Table 2 | Mean myofiber diameter and fiber-type distribution in skeletal muscle biopsies pre- and post-training

\begin{tabular}{lcccc}
\hline & Pre & Post & Difference & t-test \\
\hline All fibers & & & & \\
Size $(\mu \mathrm{m})$ & $49.6 \pm 15.6$ & $49.5 \pm 15.8$ & $-0.3 \%$ & N.S \\
Fast-type fibers & & & & \\
Size $(\mu \mathrm{m})$ & $46.8 \pm 14.4$ & $47.8 \pm 15.8$ & $+2.2 \%$ & $\boldsymbol{p}<\mathbf{0 . 0 0 0 1}$ \\
Percentage & $45 \%$ & $49 \%$ & $+8.9 \%$ & N.S \\
Slow-type fibers & & & & \\
Size $(\mu \mathrm{m})$ & $50.4 \pm 14.8$ & $48.4 \pm 16.7$ & $-3.6 \%$ & $\boldsymbol{p}<\mathbf{0 . 0 0 0 1}$ \\
Percentage & $55 \%$ & $51 \%$ & $-7.2 \%$ & N.S \\
\hline
\end{tabular}

The overall mean myofiber diameter did not significantly change after 9 weeks of training, while a significant increase of fast-type mean myofiber diameter was observed. Values are given in mean $\pm S D$. Bold font indicates statistically significant values. N.S, No statistically significant.

To support this evidence, we analyzed the expression of miR-29, which controls the extracellular proteins and the fibrotic process (van Rooij et al., 2008; Cacchiarelli et al., 2010; He et al., 2013). qRT-PCR revealed that ES promotes a significant increase in miR29 expression (Figure 4), suggesting that mir-29 controls fibrosis in ES stimulated muscle.

\section{DISCUSSION}

Electrical stimulation has been proved to be very effective in restoring muscle mass and function in denervated muscles (Kern et al., 

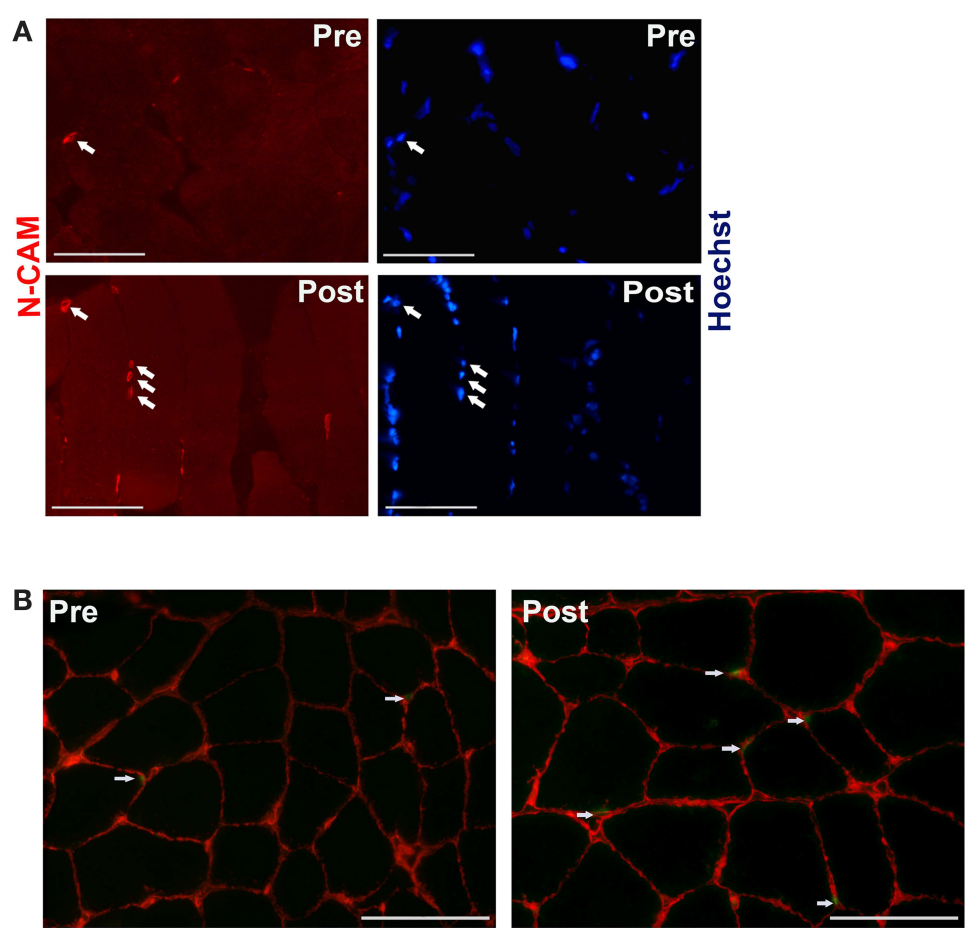

Laminin/Pax7
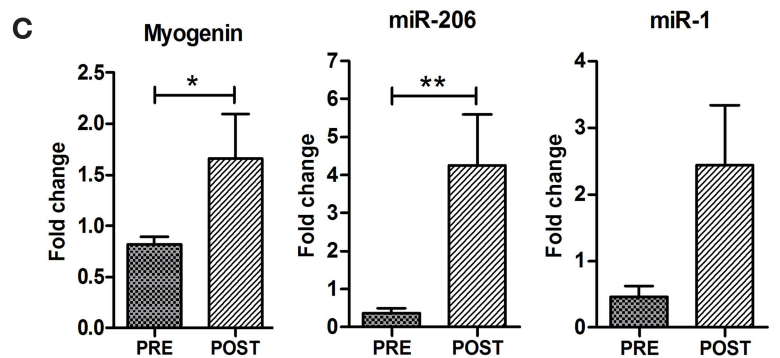

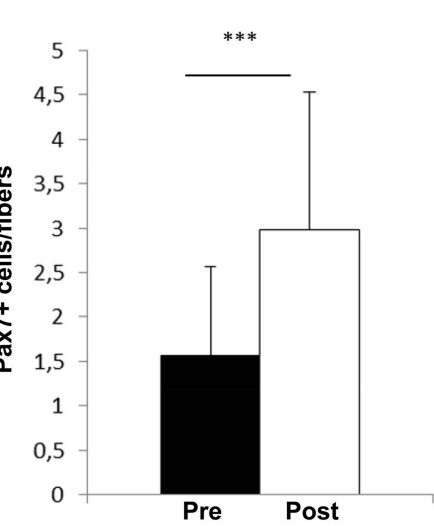

FIGURE 2 | Electrical stimulation induces an increase of satellite cells

(A) Representative immunofluorescence analysis for $\mathrm{N}$-CAM expression (red stained, arrowed). N-CAM expressing cells are increased in post-trained muscle compared with the pre-training condition. Nuclei are counterstained in blue with Hoechst. Bar $100 \mu \mathrm{m}$. (B) Representative co-immunofluorescence analyses of laminin (red staining) and Pax7 (green staining) expression in skeletal muscle biopsies comparing pre- to post-training conditions. The number of Pax7 positive cells (arrowed) is increased in biopsies of post-trained subjects, compared to the pre-training ones. Bar $100 \mu \mathrm{m}$. Right panel: percentage of Pax7+ cells in pre-trained and post-ES-trained muscles. Data are represented as average $\pm \mathrm{SD}$. ${ }^{* *} p<0.0001$. (C) Real time PCR analysis for myogenin, miR-206, and miR-1 expression in pre-trained (PRE) and post-ES-trained (POST) muscles. Data are represented as average \pm SEM. $n=16 .{ }^{*} p<0.05 ;{ }^{*} p<0.005$.
2004,2008, 2010; Carraro et al., 2005; Ashley et al., 2007; Maddocks et al., 2013).

The aim of the present study was to verify whether ES can be used to improve muscle function in elderly individuals. It is well documented that training and regular exercise can attenuate the pathological signs of sarcopenia, increasing muscle strength while decreasing fall risk. Nevertheless, certain pathologic conditions (e.g., sarcopenia, osteoarthritis, disuse associated atrophy, muscular dystrophies, trauma, and injuries) limit the ability to perform physical exercise. An alternative effective intervention to improve muscle recovery is ES.

We evaluated the functional performance of ES-trained subject and analyzed the molecular signature of ES-mediated effects on skeletal muscle. In our study, we did not include untreated controls but we compared the functional performance of same subjects before and after ES training.

The results collected here suggest that ES, similarly to physical exercise, attenuate the functional decline associated with aging, improving muscle strength and mass, maintaining the overall size of muscle fibers (decreasing during aging), activating satellite cell, and guaranteeing muscle adaptation. Thus, ES should be protective for sarcopenia.

All functional tests and maximal isometric torque showed significant improvements after 9 weeks of ES training, counteracting age-depended mobility ability, frailty, risk of falling, while improving functional performance and ADL. Of note, the ES-treated 

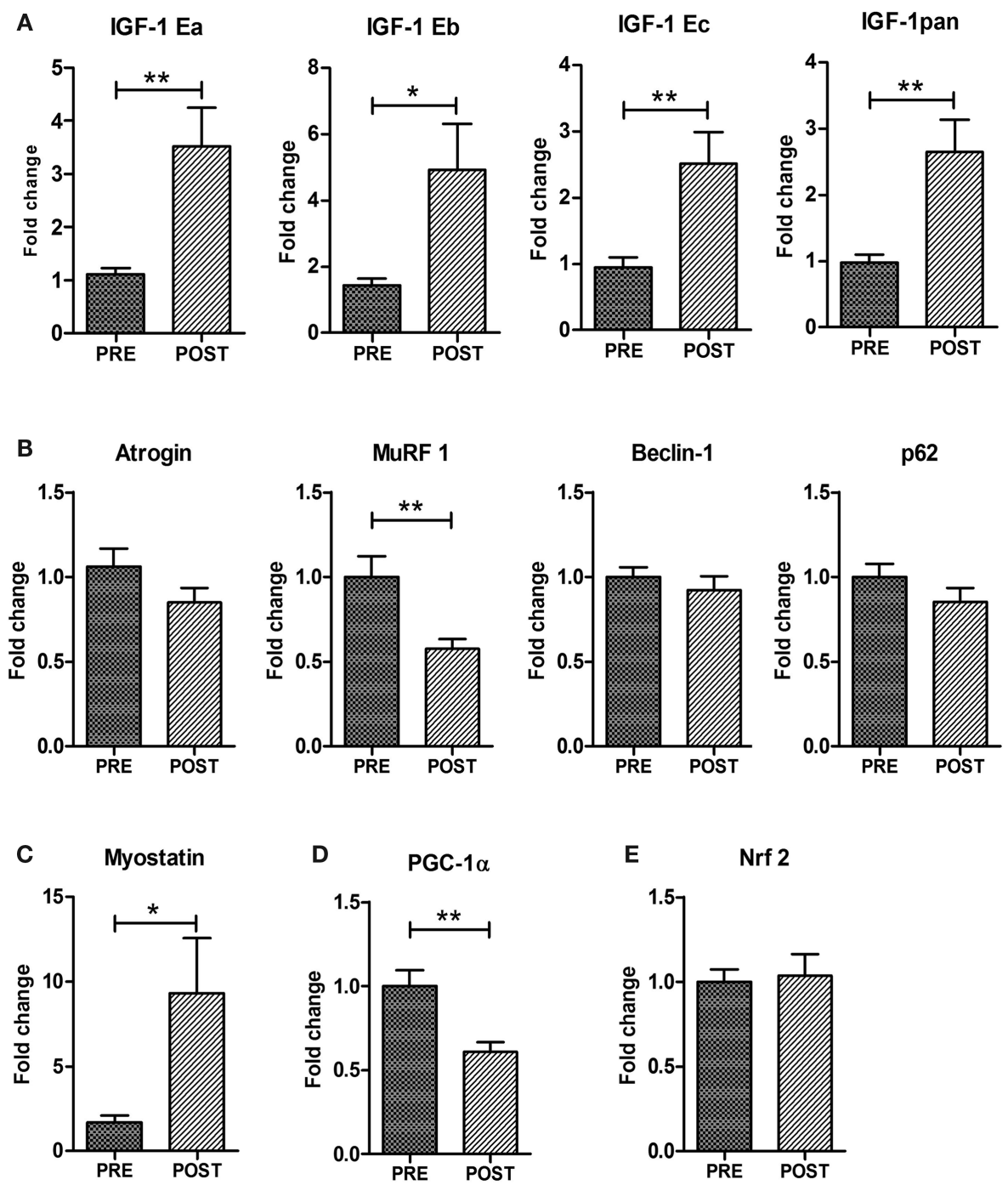

FIGURE 3 | Expression analyses of genes controlling muscle mass and metabolism. Real time PCR analysis for the expression of IGF-1 isoforms (total IGF-1 pan, IGF-1Ea, IGF-1Eb, IGF-1Ec) (A) Atrogin-1, MurF-1, Beclin1, p62 (B), Myostatin (C), PGC1 $\alpha$ (D), and Nrf2 (E). Data are represented as average \pm SEM. $n=16$. ${ }^{*} p<0.005 ;{ }^{*}{ }^{*} p<0.0005$

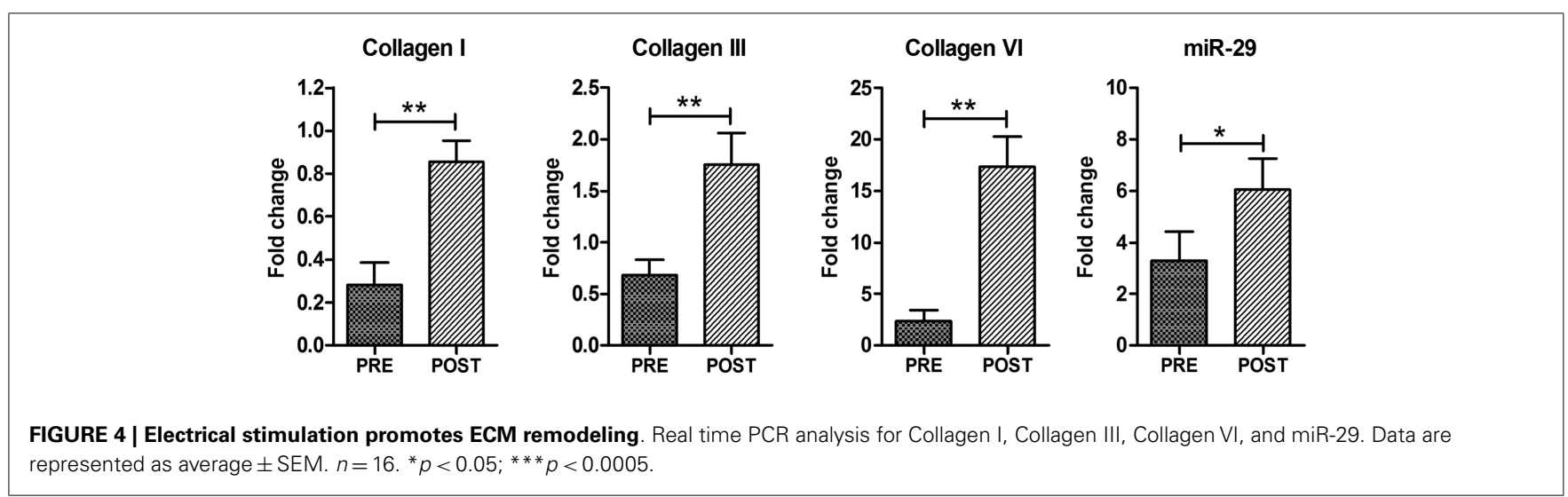


subjected performed similarly to a 6-week resistance trained older adults (70.6 \pm 6.1 years) (Fragala et al., 2014), assessed with TUGT, $5 \times$ Chair Rise and gait time.

It has been reported that female subjects displayed higher sensory and supramotor excitability to surface ES (Maffiuletti et al., 2008). In our study, the intensity (current) was very similar in all (male and female) subjects, which were instructed to increase the stimulation intensity until their maximum sensory tolerance level was reached. None of them declared problematic events during training sessions, and both males and females reported slight pain clinically not relevant at rest before ES, without changes through ES training. In the functional tests, the males were generally stronger and faster than females except chair rise (females faster) and stair test (equal). Both males and females improved in all tests but females gained in percentage nearly twice as males. This could be explained taking into consideration that the electrodes we used in our study covered a relative larger area of the thigh in female than male and therefore activated more motorpoints by stimulation. This results in training of a larger volume of the quadriceps muscles similar to the observation reported by Maffiuletti et al. (2008). We suggest, for all therapeutic applications of ES that aim to improve force and quality of muscle structure, to use electrode sizes greater than $100 \mathrm{~cm}^{2}$ to cover larger portions of the muscle and at the same time to create less discomfort due to low current density at the electrode-skin interface (Kern, 2014).

At molecular level, we demonstrated that ES promotes the modulation of factors associated with muscle growth and induces a remodeling of ECM. Our findings demonstrated that ES of 73.1 years old healthy sedentary seniors, increases expression of IGF-1 and of relevant biomarkers of activated satellite cells and myoblasts, reduces expression of muscle-specific atrophy-related ubiquitin ligase genes, and promotes the remodeling of myofibers and of ECM.

IGF-1 plays an important anabolic role in skeletal muscle and it is an important modulator of muscle growth and regeneration. Different evidences indicate that, during muscle regeneration, IGF-IEb levels is responsible for activating and for proliferating satellite cells; IGF-1Ea is responsible for myoblast differentiation and IGF-1Ec expression is normally up-regulated in response to mechanical signals (Matheny et al., 2010). Thus, our data suggests that ES stimulates the expression of different isoforms of IGF-1 in muscle, guaranteeing muscle homeostasis and protection against age-related sarcopenia. In fact, increased levels of IGF-1 were associated with a reduced level of expression of MuRF-1, a gene involved in muscle atrophy.

Among potential molecular mechanisms activated by exercise, autophagy might play a critical role for metabolic adaptation (Lira et al., 2013; Ferraro et al., 2014; Vainshtein et al., 2014). In particular, it has been reported that autophagy is an essential process for skeletal muscle adaptation and physical performance after endurance training (Lira et al., 2013). Conversely, we did not observe significant activation of autophagic pathway in ES-treated subjects. This can be justified considering that the up-regulation of autophagic-relevant markers is an early event and then they returned to basal levels shortly after the stimulus. We analyzed the muscle biopsies 7 days after the last ES treatments, a time point that might not sustain the activation of autophagic pathways. Further analysis will clarify and address this point.

Of note, myostatin was up-regulated in ES-treated muscles. We can interpret this result considering that myostatin may be produced locally by skeletal muscle cells to limit the muscle growth stimulated by IGF-1, guaranteeing an appropriate organ size (Shyu et al., 2005).

The up-regulation of myostatin can be also explained considering that ES guarantees a balance between satellite cells activation and differentiation. In fact, skeletal muscle differentiation is a complex and highly regulated process characterized by morphological changes, which include myoblast proliferation, alignment, elongation, and fusion into multinucleated myotubes. This is a balanced process dynamically coordinated by positive and negative signals. Recent studies revealed that IGF-1 also stimulates the expression of myostatin and it has been suggested that myostatin and IGF-1 positively coordinate myogenesis (Kurokawa et al., 2009; Valdés et al., 2013). Interestingly, it has been recently reported that myostatin stimulates $\mathrm{C} 2 \mathrm{C} 12$ proliferation, and this effect occurred in the presence of IGF-1 (Rodgers et al., 2014). Thus, it is possible that in our experimental model the modulation of myostatin is independent by miR-206 expression/activity; however, myostatin and the relevant markers of activated and differentiating satellite cells are part of the mechanism for muscle adaptation induced by ES.

Interestingly, the up-regulation of collagen VI and ECM remodeling suggests that ES strengths key component of the satellite cell niche (Urciuolo et al., 2013). It has been recently suggested that ES stimulates satellite cells and a strengthened ECM, factors that are likely to be involved in protecting the muscle from damage on exposure to subsequent injuring stimuli (Mackey et al., 2011).

These results are also in agreement with morphometric analyses, which showed an increase of the percentage and diameter of the fast-type fibers.

Of clinical interest was the up-regulation of miR-29, which control fibrosis in different tissues, including skeletal muscle (Cacchiarelli et al., 2010). Considering that sarcopenia involves a decrease in muscle integrity as fibrotic invasions replace functional contractile tissue, and a progressive loss of the most powerful fast fibers, our data clearly indicate that ES improves muscle function and mass and protects against accumulation of fibrosis, regulating key factors, and signaling of muscle homeostasis and growth.

Altogether, the molecular data support our clinical findings that neuromuscular ES positively influences excitability and recruitment of stimulated muscle fibers resulting in greater force and better coordination guaranteeing, $\mathrm{ADL}$, exercise programs, and rehabilitation strategies.

In conclusion, a three times a week ES is an effective therapy to improve molecular adaptations of muscle, counteracting muscle atrophy, and improving functional outcomes with positive influence on quality of life of seniors.

\section{AUTHOR CONTRIBUTIONS}

Helmut Kern, Samantha Burggraf, Nejc Sarabon, Matthias Krenn, Jan Cvecka: designed the clinical work, recruited senior subjects and made clinical evaluation, drafted the work, reviewed 
the work. Helmut Kern, Stefan Löfler, Michael Vogelauer, Winfried Mayr, Hannah Fruhmann: collected human biopsies and samples; designed the ES protocol, performed functional evaluation on senior subjects, acquisition, statistical analysis, and interpretation of clinical data, drafted the work, reviewed the work. Laura Barberi, Simona Sbardella, Vanina Romanello, Marco Sandri, Antonio Musaro: perform molecular analysis, acquisition, statistic analysis, and interpretation of gene expression data, reviewed the work. Laura Pietrangelo, Feliciano Protasi: performed electron microscopy analysis, measured frequency, and position of CRUs and mitochondria, acquisition, statistical analysis, and interpretation of data, drafted the work, reviewed the work. Sandra Zampieri, Ugo Carraro: performed histological analysis, acquisition, statistic analysis, and interpretation of data; drafted the work, reviewed the work. Antonio Musaro, Marco Sandri, Helmut Kern, Ugo Carraro, Feliciano Protasi, Sandra Zampieri: designed and organized the experiments, interpreted the results, critically revised the work. Antonio Musaro: wrote the paper. All authors approved the final version of the manuscript.

\section{ACKNOWLEDGMENTS}

This study was supported by PRIN; European Regional Development Fund-Cross Border Cooperation Programme SLOVAKIAAUSTRIA (Interreg-Iva) project "Mobilität im Alter" MOBIL N_00033; Austrian Federal Ministry of Science and Research; Ludwig Boltzmann Society (Vienna); Telethon (GGP13013 to Antonio Musaro and 13213 to Feliciano Protasi); AFM; ASI.

\section{REFERENCES}

Adamo, M. L., and Farrar, R. P. (2006). Resistance training, and IGF involvement in the maintenance of muscle mass during the aging process. Ageing Res. Rev. 5, 310-331. doi:10.1016/j.arr.2006.05.001

Alnaqeeb, M. A., and Goldspink, G. (1987). Changes in fibre type, number and diameter in developing and ageing skeletal muscle. J. Anat. 153, 31-45.

Ashley, Z., Salmons, S., Boncompagni, S., Protasi, F., Russold, M., Lanmuller, H., et al. (2007). Effects of chronic electrical stimulation on long-term denervated muscles of the rabbit hind limb. J. Muscle Res. Cell. Motil. 28, 203-217. doi:10.1007/s10974-007-9119-4

Bax, L., Staes, F., and Verhagen, A. (2005). Does neuromuscular electrical stimulation strengthen the quadriceps femoris? A systematic review of randomised controlled trials. Sports Med. 35, 191-212. doi:10.2165/00007256-200535030-00002

Berg, U., and Bang, P. (2004). Exercise and circulating insulin-like growth factor I. Horm. Res. 62, 50-58. doi:10.1159/000080759

Biral, D., Kern, H., Adami, N., Boncompagni, S., Protasi, F., and Carraro, U. (2008). Atrophy-resistant fibers in permanent peripheral denervation of human skeletal muscle. Neurol. Res. 30, 137-144. doi:10.1179/174313208X281145

Bohannon, R. W. (1997). Isometric strength data. Arch. Phys. Med. Rehabil. 78, 566-567. doi:10.1016/S0003-9993(97)90181-7

Bohannon, R. W., and Williams Andrews, A. (2011). Normal walking speed: a descriptive meta-analysis. Physiotherapy 97, 182-189. doi:10.1016/j.physio.2010

Boncompagni, S., Kern, H., Rossini, K., Hofer, C., Mayr, W., Carraro, U., et al. (2007). Structural differentiation of skeletal muscle fibers in the absence of innervation in humans. Proc. Natl. Acad. Sci. U.S.A. 104, 19339-19344. doi:10.1073/pnas.0709061104

Cacchiarelli, D., Martone, J., Girardi, E., Cesana, M., Incitti, T., Morlando, M., et al. (2010). MicroRNAs involved in molecular circuitries relevant for the Duchenne muscular dystrophy pathogenesis are controlled by the dystrophin/nNOS pathway. Cell Metab. 12, 341-351. doi:10.1016/j.cmet.2010.07.008

Carosio, S., Berardinelli, M. G., Aucello, M., and Musarò, A. (2011). Impact of ageing on muscle cell regeneration. Ageing Res. Rev. 10, 35-42. doi:10.1016/j.arr.2009. 08.001
Carraro, U., Rossini, K., Mayr, W., and Kern, H. (2005). Muscle fiber regeneration in human permanent lower motoneuron denervation: relevance to safety and effectiveness of ES-training, which induces muscle recovery in SCI subjects. Artif. Organs 29, 187-191. doi:10.1111/j.1525-1594.2005.29032.x

Chen, J. F., Mandel, E. M., Thomson, J. M., Wu, Q., Callis, T. E., Hammond, S. M., et al. (2006). The role of microRNA-1 and microRNA-133 in skeletal muscle proliferation and differentiation. Nat. Genet. 38, 228-233. doi:10.1038/ ng1725

Clop, A., Marcq, F., Takeda, H., Pirottin, D., Tordoir, X., Bibé, B., et al. (2006). A mutation creating a potential illegitimate microRNA target site in the myostatin gene affects muscularity in sheep. Nat. Genet. 38, 813-818. doi:10.1038/ng1810

Corrigan, D., and Bohannon, R. W. (2001). Relationship between knee extension force and stand-up performance in community-dwelling elderly women. Arch. Phys. Med. Rehabil. 82, 1666-1672. doi:10.1053/apmr.2001.26811

Cruz-Jentoft, A. J. (2013). Perspective: protein and exercise for frailty and sarcopenia: still learning. J. Am. Med. Dir. Assoc. 14, 69-71. doi:10.1016/j.jamda.2012.09.024

de Oliveira Melo, M., Aragão, F. A., and Vaz, M. A. (2013). Neuromuscular electrical stimulation for muscle strengthening in elderly with knee osteoarthritis - a systematic review. Complement. Ther. Clin. Pract. 19, 27-31. doi:10.1016/j.ctcp. 2012.09.002

Elliott, B., Renshaw, D., Getting, S., and Mackenzie, R. (2012). The central role of myostatin in skeletal muscle and whole body homeostasis. Acta Physiol. (Oxf.) 205, 324-340. doi:10.1111/j.1748-1716.2012.02423.x

Ferraro, E., Giammarioli, A. M., Chiandotto, S., Spoletini, I., and Rosano, G. (2014). Exercise-induced skeletal muscle remodeling and metabolic adaptation: redox signaling and role of autophagy. Antioxid. Redox Signal. 21, 154-176. doi:10.1089/ars.2013.5773

Fragala, M. S., Fukuda, D. H., Stout, J. R., Townsend, J. R., Emerson, N. S., Boone, C. H., et al. (2014). Muscle quality index improves with resistance exercise training in older adults. Exp. Gerontol. 53, 1-6. doi:10.1016/j.exger.2014.01.027

Freiberger, E., Blank, W. A., Salb, J., Geilhof, B., Hentschke, C., Landendoerfer, P., et al. (2013). Effects of a complex intervention on fall risk in the general practitioner setting: a cluster randomized controlled trial. Clin. Interv. Aging 8, 1079-1088. doi:10.2147/CIA.S46218

Giggins, O., Fullen, B., and Coughlan, G. (2012). Neuromuscular electrical stimulation in the treatment of knee osteoarthritis: a systematic review and metaanalysis. Clin. Rehabil. 26, 867-881. doi:10.1177/0269215511431902

Guralnik, J. M., Ferrucci, L., Pieper, C. F., Leveille, S. G., Markides, K. S., Ostir, G. V., et al. (2000). Lower extremity function and subsequent disability: consistency across studies, predictive models, and value of gait speed alone compared with the short physical performance battery. J. Gerontol. A Biol. Sci. Med. Sci. 55, M221-M231. doi:10.1093/gerona/55.4.M221

Guralnik, J. M., Simonsick, E. M., Ferrucci, L., Glynn, R. J., Berkman, L. F., Blazer, D. G., et al. (1994). A short physical performance battery assessing lower extremity function: association with self-reported disability and prediction of mortality and nursing home admission. J. Gerontol. 49, M85-M94. doi:10.1093/geronj/49.2.M85

He, Y., Huang, C., Lin, X., and Li, J. (2013). MicroRNA-29 family, a crucial therapeutic target for fibrosis diseases. Biochimie 95, 1355-1359. doi:10.1016/j.biochi. 2013.03.010

Kadi, F., Eriksson, A., Holmner, S., Butler-Browne, G. S., and Thornell, L. E. (1999) Cellular adaptation of the trapezius muscle in strength-trained athletes. Histochem. Cell Biol. 111, 189-195. doi:10.1007/s004180050348

Kern, H. (2014). Functional electrical stimulation on paraplegic patients. Eur. J. Transl. Myol. 24, 75-157. doi:10.4081/bam.2014.2.75

Kern, H., Boncompagni, S., Rossini, K., Mayr, W., Fanò, G., Zanin, M. E., et al. (2004). Long-term denervation in humans causes degeneration of both contractile and excitation-contraction coupling apparatus that can be reversed by functional electrical stimulation (ES). A role for myofiber regeneration? J. Neuropathol. Exp. Neurol. 63, 919-931.

Kern, H., Carraro, U., Adami, N., Biral, D., Hofer, C., Forstner, C., et al. (2010). Home-based functional electrical stimulation rescues permanently denervated muscles in paraplegic patients with complete lower motor neuron lesion. $\mathrm{Neu}$ rorehabil. Neural Repair 24, 709-721. doi:10.1177/1545968310366129

Kern, H., Hofer, C., Mödlin, M., Mayr, W., Vindigni, V., Zampieri, S., et al. (2008). Stable muscle atrophy in long-term paraplegics with complete upper motor neuron lesion from 3- to 20-year SCI. Spinal Cord 46, 293-304. doi:10.1038/sj. sc. 3102131 
Kern, H., Pelosi, L., Coletto, L., Musarò, A., Sandri, M., Vogelauer, M., et al. (2011). Atrophy/hypertrophy cell signaling in muscles of young athletes trained with vibrational-proprioceptive stimulation. Neurol. Res. 33, 998-1009. doi:10.1179/ $016164110 X 12767786356633$

Krenn, M., Haller, M., Bijak, M., Unger, E., Hofer, C., Kern, H., et al. (2011). Safe neuromuscular electrical stimulator designed for the elderly. Artif. Organs 35, 253-256. doi:10.1111/j.1525-1594.2011.01217.x

Kurokawa, M., Sato, F., Aramaki, S., Soh, T., Yamauchi, N., and Hattori, M. A. (2009). Monitor of the myostatin autocrine action during differentiation of embryonic chicken myoblasts into myotubes: effect of IGF-I. Mol. Cell. Biochem. 331, 193-199. doi:10.1007/s11010-009-0158-6

Levine, M., McElroy, K., Stakich, V., and Cicco, J. (2013). Comparing conventional physical therapy rehabilitation with neuromuscular electrical stimulation after TKA. Orthopedics 36, e319-e324. doi:10.3928/01477447-20130222-20

Lin, J., Wu, H., Tarr, P. T., Zhang, C. Y., Wu, Z., Boss, O., et al. (2002). Transcriptional co-activator PGC-1 alpha drives the formation of slow-twitch muscle fibres. Nature 418, 797-801. doi:10.1038/nature00904

Lira, V. A., Okutsu, M., Zhang, M., Greene, N. P., Laker, R. C., Breen, D. S., et al. (2013). Autophagy is required for exercise training-induced skeletal muscle adaptation and improvement of physical performance. FASEB J. 27, 4184-4193. doi:10.1096/fj.13-228486

Livak, K. J., and Schmittgen, T. D. (2001). Analysis of relative gene expression data using real-time quantitative PCR and the 2(-Delta Delta $\mathrm{C}(\mathrm{T})$ ). Methods 25, 402-408. doi:10.1006/meth.2001.1262

Mackey, A. L., Brandstetter, S., Schjerling, P., Bojsen-Moller, J., Qvortrup, K., Pedersen, M. M., et al. (2011). Sequenced response of extracellular matrix deadhesion and fibrotic regulators after muscle damage is involved in protection against future injury in human skeletal muscle. FASEB J. 25, 1943-1959. doi:10.1096/fj.10-176487

Maddocks, M., Gao, W., Higginson, I. J., and Wilcock, A. (2013). Neuromuscular electrical stimulation for muscle weakness in adults with advanced disease. Cochrane Database Syst. Rev. 1, 1-30. doi:10.1002/14651858.CD009419.pub2

Maffiuletti, N. A., Herrero, A. J., Jubeau, M., Impellizzeri, F. M., and Bizzini, M. (2008). Differences in electrical stimulation thresholds between men and women. Ann. Neurol. 63, 507-512. doi:10.1002/ana.21346

Matheny, R. W. Jr, Nindl, B. C., and Adamo, M. L. (2010). Minireview: mechanogrowth factor: a putative product of IGF-I gene expression involved in tissue repair and regeneration. Endocrinology 151, 865-875. doi:10.1210/en.2009-1217

Mosole, S., Carraro, U., Kern, H., Loefler, S., Fruhmann, H., Vogelauer, M., et al. (2014). Long-term high-level exercise promotes muscle reinnervation with age. J. Neuropathol. Exp. Neurol. 73, 284-294. doi:10.1097/NEN.0000000000000032

Musarò, A., McCullagh, K., Paul, A., Houghton, L., Dobrowolny, G., Molinaro, M., et al. (2001). Localized Igf-1 transgene expression sustains hypertrophy and regeneration in senescent skeletal muscle. Nat. Genet. 27, 195-200. doi:10.1038/ 84839

Nuhr, M. J., Pette, D., Berger, R., Quittan, M., Crevenna, R., Huelsman, M., et al. (2004). Beneficial effects of chronic low-frequency stimulation of thigh muscles in patients with advanced chronic heart failure. Eur. Heart J. 25, 136-143. doi:10.1016/j.ehj.2003.09.027

Paffenbarger, R. S. Jr, Kampert, J. B., Lee, I. M., Hyde, R. T., Leung, R. W., and Wing, A. L. (1994). Changes in physical activity and other lifeway patterns influencing longevity. Med. Sci. Sports Exerc. 26, 857-865. doi:10.1249/00005768199407000-00008

Podsiadlo, D., and Richardson, S. (1991). The timed "Up \& Go": a test of basic functional mobility for frail elderly persons. J. Am. Geriatr. Soc. 39, 142-148.

Quittan, M., Wiesinger, G. F., Sturm, B., Puig, S., Mayr, W., Sochor, A., et al. (2001). Improvement of thigh muscles by neuromuscular electrical stimulation in patients with refractory heart failure: a single-blind, randomized, controlled trial. Am. J. Phys. Med. Rehabil. 80, 206-214. doi:10.1097/00002060-200103000-00011

Rao, P. K., Kumar, R. M., Farkhondeh, M., Baskerville, S., and Lodish, H. F. (2006). Myogenic factors that regulate expression of muscle-specific microRNAs. Proc. Natl. Acad. Sci. U.S.A. 103, 8721-8726. doi:10.1073/pnas.0602831103

Rejeski, W. J., Ettinger, W. H. Jr, Schumaker, S., James, P., Burns, R., and Elam, J. T. (1995). Assessing performance-related disability in patients with knee osteoarthritis. Osteoarthr. Cartil. 3, 157-167. doi:10.1016/S1063-4584(05) 80050-0

Rodgers, B. D., Wiedeback, B. D., Hoversten, K. E., Jackson, M. F., Walker, R. G., and Thompson, T. B. (2014). Myostatin stimulates, not inhibits, C2C12 myoblast proliferation. Endocrinology 155, 670-675. doi:10.1210/en.2013-2107
Rosemffet, M. G., Schneeberger, E. E., Citera, G., Sgobba, M. E., Laiz, C., Schmulevich, H., et al. (2004). Effects of functional electrostimulation on pain, muscular strength, and functional capacity in patients with osteoarthritis of the knee. J. Clin. Rheumatol. 10, 246-249. doi:10.1097/01.rhu.0000141831. 40350.91

Rossini, K., Zanin, M. E., Podhorska-Okolow, M., and Carraro, U. (2002). To stage and quantify regenerative myogenesis in human long-term permanent denervated muscle. Basic Appl. Myol. 12, 277-286.

Šarabon, N., Loefler, S., Cvecka, J., Sedliak, M., and Kern, H. (2013a). Strength training in elderly people improves static balance: a randomized controlled trial. Eur. J. Transl. Myol. 23, 85-89. doi:10.4081/bam.2013.3.85

Šarabon, N., Rošker, J., Fruhmann, H., Burggraf, S., Loefler, S., and Kern, H. (2013b). Reliability of maximal voluntary contraction related parameters measured by a portable isometric knee dynamometer. Phys. Med. Rehabil. Kuror. 23, 22-27. doi:10.1055/s-0032-1331190

Šarabon, N., Loefler, S., Fruhmann, H., Burggraf, S., and Kern, H. (2010a). Reduction and technical simplification of testing protocol for walking based on repeatability analyses: an Interreg IVa pilot study. Eur. J. Transl. Myol. 1, 181-186. doi:10.4081/bam.2010.4.181

Šarabon, N., Rošker, J., Loefler, S., and Kern, H. (2010b). Sensitivity of body sway parameters during quiet standing to manipulation of support surface size. J. Sport Sci. Med. 9, 431-438.

Scicchitano, B. M., Rizzuto, E., and Musarò, A. (2009). Counteracting muscle wasting in aging and neuromuscular diseases: the critical role of IGF-1. Aging (Albany NY) 1, 451-457.

Shyu, K. G., Ko, W. H., Yang, W. S., Wang, B. W., and Kuan, P. (2005). Insulinlike growth factor-1 mediates stretch-induced upregulation of myostatin expression in neonatal rat cardiomyocytes. Cardiovasc. Res. 68, 405-414. doi:10.1016/ j.cardiores.2005.06.028

Snijders, T., Verdijk, L. B., and van Loon, L. J. (2009). The impact of sarcopenia and exercise training on skeletal muscle satellite cells. Ageing Res. Rev. 8, 328-338. doi:10.1016/j.arr.2009.05.003

Strasser, E. M., Stättner, S., Karner, J., Klimpfinger, M., Freynhofer, M., Zaller, V., et al. (2009). Neuromuscular electrical stimulation reduces skeletal muscle protein degradation and stimulates insulin-like growth factors in an ageand current-dependent manner: a randomized, controlled clinical trial in major abdominal surgical patients. Ann. Surg. 249, 738-743. doi:10.1097/SLA. 0b013e3181a38e71

Suzuki, T., Bean, J. F., and Fielding, R. A. (2001). Muscle power of the ankle flexors predicts functional performance in community-dwelling older women. J. Am. Geriatr. Soc. 49, 1161-1167. doi:10.1046/j.1532-5415.2001.49232.x

Urciuolo, A., Quarta, M., Morbidoni, V., Gattazzo, F., Molon, S., Grumati, P., et al. (2013). Collagen VI regulates satellite cell self-renewal and muscle regeneration. Nat. Commun. 4, 1964-1989. doi:10.1038/ncomms2964

Vainshtein, A., Grumati, P., Sandri, M., and Bonaldo, P. (2014). Skeletal muscle, autophagy, and physical activity: the ménage à trois of metabolic regulation in health and disease. J. Mol. Med. 92, 127-137. doi:10.1007/s00109-013-1096-z

Valdés, J. A., Flores, S., Fuentes, E. N., Osorio-Fuentealba, C., Jaimovich, E., and Molina, A. (2013). IGF-1 induces IP3-dependent calcium signal involved in the regulation of myostatin gene expression mediated by NFAT during myoblast differentiation. J. Cell. Physiol. 228, 1452-1463. doi:10.1002/jcp.24298

van Rooij, E., Liu, N., and Olson, E. N. (2008). MicroRNAs flex their muscles. Trends Genet. 24, 159-166. doi:10.1016/j.tig.2008.01.007

Viana, J. U., Silva, S. L., Torres, J. L., Dias, J. M., Pereira, L. S., and Dias, R. C. (2013). Influence of sarcopenia and functionality indicators on the frailty profile of community-dwelling elderly subjects: a cross-sectional study. Braz. J. Phys. Ther. 17, 373-381. doi:10.1590/S1413-35552012005000102

Vinciguerra, M., Musaro, A., and Rosenthal, N. (2010). Regulation of muscle atrophy in aging and disease. Adv. Exp. Med. Biol. 694, 211-233. doi:10.1007/978-14419-7002-2_15

Walker, D. K., Fry, C. S., Drummond, M. J., Dickinson, J. M., Timmerman, K. L., Gundermann, D. M., et al. (2012). PAX7+ satellite cells in young and older adults following resistance exercise. Muscle Nerve 46, 51-59. doi:10.1002/mus.23266

Wall, B. T., Dirks, M. L., Verdijk, L. B., Snijders, T., Hansen, D., Vranckx, P., et al. (2012). Neuromuscular electrical stimulation increases muscle protein synthesis in elderly type 2 diabetic men. Am. J. Physiol. Endocrinol. Metab. 303, 614-623. doi:10.1152/ajpendo.00138.2012

Zampieri, S., Doria, A., Adami, N., Biral, D., Vecchiato, M., Savastano, S., et al. (2010). Subclinical myopathy in patients affected with newly diagnosed colorectal cancer 
at clinical onset of disease: evidence from skeletal muscle biopsies. Neurol. Res. 32, 20-25. doi:10.1179/016164110X12556180205997

Zampieri, S., Pietrangelo, L., Loefler, S., Fruhmann, H., Vogelauer, M., Burggraf, S., et al. (2014). Lifelong physical exercise delays age-associated skeletal muscle decline. J. Gerontol. A Biol. Sci. Med. Sci. doi:10.1093/gerona/glu006

Conflict of Interest Statement: The review process was handled objectively despite Luciano Merlini having collaborated with the authors. The authors declare that the research was conducted in the absence of any commercial or financial relationships that could be construed as a potential conflict of interest.

Received: 22 April 2014; paper pending published: 19 June 2014; accepted: 10 July 2014; published online: 24 July 2014.
Citation: Kern H, Barberi L, Löfler S, Sbardella S, Burggraf S, Fruhmann H, Carraro U, Mosole S, Sarabon N, Vogelauer M, Mayr W, Krenn M, Cvecka J, Romanello V, Pietrangelo L, Protasi F, Sandri M, Zampieri S and Musaro A (2014) Electrical stimulation counteracts muscle decline in seniors. Front. Aging Neurosci. 6:189. doi: 10.3389/fnagi.2014.00189

This article was submitted to the journal Frontiers in Aging Neuroscience.

Copyright (c) 2014 Kern, Barberi, Löfler, Sbardella, Burggraf, Fruhmann, Carraro, Mosole, Sarabon, Vogelauer, Mayr, Krenn, Cvecka, Romanello, Pietrangelo, Protasi, Sandri, Zampieri and Musaro. This is an open-access article distributed under the terms of the Creative Commons Attribution License (CC BY). The use, distribution or reproduction in other forums is permitted, provided the original author(s) or licensor are credited and that the original publication in this journal is cited, in accordance with accepted academic practice. No use, distribution or reproduction is permitted which does not comply with these terms. 